\title{
Factores de riesgo en la violencia contra la mujer en el municipio de Ventaquemada (Boyacá) desde una mirada de trabajo social
}

\section{Risk factors in violence against women in the municipality of Ventaquemada (Boyacá) from a perspective of Social Work}

\author{
María Tatiana Suárez-Sierra \\ $\bowtie$ mtsuarez@jdc.edu.co \\ Fundación Universitaria Juan de Castellanos Programa de Trabajo Social, \\ Facultad de Ciencias Sociales y Económicas, , Tunja, Boyacá, Colombia
}

Recibido: $18-02-2018$

Aceptado: 29-05-2018

Publicado: 05-06-2018

\section{RESUMEN}

Objetivo. La presente investigación tuvo como objetivo identificar los factores de riesgo que influyen en la violencia contra la mujer en el municipio de Ventaquemada (Boyacá). Asimismo, determinar los tipos de violencia ejercidos hacia este género. Método. La metodología utilizada es el enfoque cualitativo descriptivo. Se tomó una muestra de quince mujeres en situación de violencia, pertenecientes a las veredas Boquerón, El Carmen y Frutillo, seleccionadas mediante un muestreo por redes. Las técnicas aplicadas fueron la encuesta social y entrevista semiestructurada. Resultados. A partir de una lectura desde trabajo social, se estudió la teoría ecológica de los sistemas de Uriel Bronfenbrenner, en la que se determinó que una de las causas relacionadas es la de los factores individuales, relacionales y sociales, en los que se ubica el déficit económico, la alta influencia del medio y la baja autonomía, liderazgo y participación, lo que da paso a que esta población se enfrente a dinámicas de violencia física, económica, verbal, psicológica o sexual, que ocasionan daños y cambios en el ciclo vital de la mujer. Conclusiones. La normalización de la violencia contra la mujer en las veredas Boquerón, El Carmen y Frutillo del municipio de Ventaquemada se determina como uno de los factores de riesgo más relevantes.

Palabras clave: factores de riesgo, violencia contra la mujer, influencia, dinámicas, normalización. 


\section{ABSTRACT}

Objetive. The objective of this research was to identify the risk factors that influence violence against women in the municipality of Ventaquemada (Boyacá). Also, determine the types of violence exercised towards this gender. Method. The methodology used is the descriptive qualitative approach. A sample was taken of fifteen women in a situation of violence, belonging to the villages of Boquerón, El Carmen and Frutillo, selected through network sampling. Result. The techniques applied were the social survey and semi-structured interview. From a reading from social work, the ecological theory of the systems of Uriel Bronfenbrenner was studied, in which it was determined that one of the related causes is that of the individual, relational and social factors, in which the deficit is located economic, the high influence of the environment and low autonomy, leadership and participation, which gives way to this population is faced with dynamics of physical, economic, verbal, psychological or sexual violence, which cause damage and changes in the life cycle of the woman. Conclusions. The normalization of violence against women in the villages of Boquerón, El Carmen and Frutillo in the municipality of Ventaquemada is determined to be one of the most relevant risk factors.

Keywords: risk factors, violence against women, influence, dynamics, normalization.

\section{INTRODUCCIÓN}

La violencia contra la mujer se ha venido catalogando como una de las problemáticas con mayores índices a nivel mundial; se trata entonces de un fenómeno social, puesto que la Organización Mundial de la Salud estima que "el 35 por ciento de las mujeres de todo el mundo han sufrido violencia física y/o sexual por parte de su compañero sentimental" (2013, p. 1). Cabe resaltar que la mujer no solo se enfrenta a estos tipos de violencia, sino además verbal o psicológico y económica, por parte de sujetos que están inmersos en su contexto, tales como vecinos, amigos, familiares y conocidos, entre otros.

Colombia es un reproductor de violencia contra la mujer. Debemos tener en cuenta las dinámicas sociales en las que se encuentra inmersa la nación; algunos de los determinantes para esta vulneración hacia el género son el conflicto armado que ha vivido por más de cinco décadas; los imaginarios y estereotipos (Ministerio de Salud Colombiano, citado por Rodríguez, 2013) que cada comunidad tiene, y el bajo nivel de empoderamiento de la mujer, debido a que desconoce sus derechos, capacidades y habilidades, teniendo en cuenta el triple rol que esta cumple a nivel social, es decir, en el ámbito reproductivo o doméstico, productivo y comunitario.

El departamento de Boyacá está ubicado en el centro-este del país, tiene una "población de 1.278.107 personas (correspondiente al 2.7\% de la población nacional), de los cuales 637.397 son mujeres y 640.710 hombres" (DANE y Gobernación de Boyacá, 2016, p. 9). En 2016 se presentaron "1275 casos de violencias contra la mujer" (Alvarado, 2016, p. 4 ), pero debe tenerse en cuenta que no todos los actos de vulneración hacia este género han sido denunciados, esto debido a factores geográficos o por miedo e intimidación por parte del agresor. Además de esto, la mujer en el contexto boyacense se ha enfrentado a estas dinámicas por la falta de acceso al trabajo y educación, por la mínima participación social y política o por factores que el contexto va determinando. 
Este fenómeno social se ha propagado en gran magnitud en las veredas Boquerón, El Carmen y Frutillo del municipio de Ventaquemada, por lo que se realizó una investigación cualitativa descriptiva con mujeres en situación de violencia de la zona, donde se determinó desde una lectura desde trabajo social, que esta problemática está controlada por factores culturales y sociales, como la normalización de los actos de violencia hacia este género, lo que ha sido un causante de que gran parte de los casos no hayan sido denunciados.

Es por esto que desde el trabajo social como disciplina y profesión que reconoce e interviene en las dinámicas sociales que vulneran a los sujetos, surge la iniciativa investigativa de identificar y reconocer los tipos de violencia contra la mujer, ya que a partir de esto surgen orientaciones y construcciones de una política pública de equidad de género enfocada en la violencia contra la mujer y por consiguiente programas de empoderamiento e incentivación del rol de la misma en los diferentes ámbitos del contexto ventaquemense, ya que en el contexto no se había dado paso a la creación de estos mecanismos en pro de las mujeres ni a su participación activa.

Es así que entre los hallazgos más significativos de la investigación, se encontró que algunos de los determinantes de la violencia contra la mujer en las veredas Boquerón, El Carmen y Frutillo son la falta de conocimiento y presencia de diferentes redes tanto públicas como privadas; la falta de empoderamiento; la baja autoestima; el bajo nivel educativo, en virtud de que estas mujeres contrajeron matrimonio a temprana edad, y el nivel socioeconómico, debido a que la mayoría de participantes del estudio hace parte de los estratos uno y dos, es decir que son catalogadas como de nivel bajo debido a las condiciones, oportunidades y necesidades con las que cuentan. Además, deben tenerse en cuenta prácticas culturales reconocidas por las mismas mujeres, como el machismo y el sistema patriarcal que predomina en la zona.

Para el análisis de la problemática de violencia contra la mujer se estudió desde el trabajo social la teoría ecológica de los sistemas de Uriel Bronfenbrenner, ya que "el desarrollo de la persona está influenciada por el medio que lo rodea o por una serie de sistemas complejos e interactivos" (Kail y Cavanaugh, 2011, p. 10), que se pueden convertir en factores positivos o negativos en la vida de un sujeto. Esta teoría se conforma por cinco niveles: microsistema, mesosistema, exosistema, macrosistema y cronosistema, los cuales permitieron identificar cómo a partir de las influencias y dinámicas del medio, determinan acciones e imaginarios frente la violencia contra la mujer en las veredas Boquerón, El Carmen y Frutillo.

En el caso de las veredas que hacen parte del estudio se determina que el microsistema está conformado por sujetos que se encuentran en el entorno inmediato de la mujer, es decir la familia o amigos más cercanos. Cabe resaltar que de este sistema depende el ambiente de los demás, debido a la influencia que estos grupos tienen a nivel individual en la mujer agredida. Conjuntamente está el mesosistema que conecta a diversos factores inmersos en el primer subsistema, debido a que lo que suceda allí influye en la interacción de los demás medios en los cuales está inmersa la mujer, que es un factor que establece las dinámicas de esta dentro de su rol productivo, reproductivo y comunitario, así como en su condición de vulnerabilidad. 
Dentro de estos subsistemas se encuentra el exosistema, como un factor que influye indirectamente a la mujer que está inmersa en dinámicas violentas. A pesar de esto, los efectos hacia el desarrollo del individuo son evidentes porque este sistema está conformado por las situaciones sociales a las cuales se enfrenta y al contexto al cual pertenece la víctima; en este caso, el municipio de Ventaquemada (Boyacá).

El macrosistema es establecido como el enfoque más amplio al que la mujer violentada se encuentra expuesta, debido a que dentro de este subsistema están inmersas aquellas culturas y subculturas que comprenden al microsistema, mesosistema y exosistema, en el que se ha venido reconociendo por los mismos habitantes del contexto que las dinámicas en el medio son machistas y patriarcales, ya que esto se ha determinado a través del tiempo (cronosistema), mediante herencias y valores comunes que los ventaquemenses adquirieron en el contexto, los cuales se han venido modificando $y$ fortaleciendo a través del tiempo.

La violencia contra la mujer en los diferentes contextos se ha caracterizado por los actos y agresiones ejercidas, las cuales le han traído como consecuencia afectaciones a nivel psicológico, físico y social que intervienen en su rol, desempeño y desarrollo a nivel individual, familiar y dentro del medio en general. Además, como lo destacan las Naciones Unidas (1993): "La violencia contra la mujer es todo acto de violencia de género que resulte, o pueda tener como resultado un daño, ya que inclusive en este incursiona amenazas y actos de coacción o la privación arbitraria de libertad, tanto si se producen en la vida pública como en la privada" (p. 2).

Esta práctica puede reproducirse dentro de la familia o de cualquier otra relación interpersonal, ya que la mujer sufre de violencia en cualquier contexto, pero en unos es más vulnerable a estas dinámicas. Debe tenerse en cuenta que la violencia no solo se da mediante actos físicos, sino que también es ejercida mediante el lenguaje verbal y no verbal.

La violencia ejercida contra la mujer puede clasificarse de diferentes maneras: violencia física, sexual, psicológica o económica. En ellas intervienen prácticas que desequilibran el medio, limitan y generan desconocimiento del rol de este género dentro del contexto ventaquemense, disminuyen el bienestar de la mujer y por ende su calidad de vida que está enfrentada a un marco de derechos.

A partir de esto se puede determinar que la violencia física es un determinante que ha hecho que la calidad de vida, capacidades y habilidades de la mujer se vean influenciadas por el hombre, puesto que estas acciones son desarrolladas intencionalmente. Rodríguez (2013) define que:

La violencia física es la forma de agresión producida por la aplicación de la fuerza física no accidental, caracterizada por lesiones variables sobre el cuerpo de la persona agredida, con consecuencias leves o graves, que tienen efectos traumáticos de orden psicológico o emocional ya que es generada con una intención específica (p.55).

Además, la violencia física hace referencia a las agresiones recibidas por la mujer por parte de un sujeto, sea o no el cónyuge o pareja sentimental, en las cuales intervienen 
golpes, patadas, bofetadas, empujones, pellizcos, zarandeadas, también el uso de armas de fuego o cortopunzantes que pueden ocasionar la muerte. Estas acciones hacen que este género se sienta humillado, tenga miedo, presente baja autoestima, ansiedad, vergüenza, inseguridad, puesto que la mujer en la sociedad es situada en un plano inferior con respecto al hombre, y no se reconoce el triple rol que esta desarrolla en el contexto.

La violencia psicología hace referencia a agresiones verbales en las que el agresor hace uso de amenazas, humillaciones, chantajes, rechazo, burlas, insultos o palabras que tienen como fin afectar la autoestima de la mujer, desarrollar el sentimiento de inferioridad, inseguridad y subordinación. Asimismo, influye en que la mujer se aísle y tenga miedo frente sus potencialidades. Este tipo de violencia influye en la toma de decisiones, participación y autonomía de este género, puesto que genera exclusión en las diversas dinámicas presentes en la sociedad.

En este caso la violencia sexual es aquella considerada como la acción agresiva que atenta contra la intimidad, dignidad, imagen y libertad de la persona, ya que estos actos causan daños tanto físicos como emocionales, los cuales limitan la sexualidad libre y la vida reproductiva, así como en la credibilidad de este género hacia el hombre o su cónyuge, como se aprecia en el caso de las mujeres ventaquemenses. Dentro de este tipo de violencia se encuentra "la violación, forzamiento, abuso, acoso, burlas respecto a su comportamiento, insultos, agresión durante la relación, sadismo y obligación a tener relaciones cuando no se desea" (Gallardo, Gallardo, Núñez y Varela, 2009, p. 134).

Ante la violencia económica se determina que se caracteriza por la utilización de poder económico o monetario con el objetivo de dominar, subordinar y crear dependencia de la mujer hacia su pareja. Esto por medio del uso del lenguaje verbal, la destrucción de bienes y la prohibición del consumo y manejo de dinero propio. Este tipo de violencia es influenciada por los subsistemas en los cuales se desarrolla el sujeto, como en el caso de la mujer del municipio de Ventaquemada, ya que en el contexto se presentan patrones de machismo, sumisión e invisibilización del papel social de la mujer.

Al momento de determinar los tipos de violencia se tiene en cuenta que para estos existe un marco de factores de riesgo que, según el caso, son definidos como "situaciones sociales, personales o interpersonales que están presentes en los contextos donde se desarrollan los individuos y que pueden llegar a incrementar la probabilidad de que el sujeto este vulnerable" (Pérez, Barreto, Roa y Rubiano, 2015, p. 26), como la mujer de las veredas del Boquerón, El Carmen y Frutillo, quienes están expuestas a un marco de dinámicas que intervienen en que la afectación de violencia sea cada vez mayor y se normalice, sin tener en cuenta la afectación para este género, debido a condiciones, sucesos, situaciones, comportamientos o dinámicas presentes en el contexto en el que se desarrollan.

Es por esto que la mujer ventaquemense se encuentra vulnerable a diferentes factores individuales, relacionales y sociales, como lo señalan La Espada, Iraurgí y Arostegi (2004): 
Factores individuales. referidos a las características internas del individuo, frente a su forma de ser, sentirse y comportarse. Asimismo, a la edad, personalidad, habilidades o recursos sociales de que dispone, actitudes, creencias y valores, autoconcepto y autoestima, autocontrol y experimentación.

Factores relacionales. relativos al entorno más próximo de la persona. La interacción específica de cada sujeto con la familia, los amigos o grupo de pares, contexto escolar y ocio, noche, fines de semana, diversión, dinero.

Factores sociales. se vinculan a un entorno social más amplio. La estructura económica, normativa, accesibilidad y disponibilidad de la mujer hacia su medio, aceptación social del mismo, costumbres y tradiciones características de unas culturas que afectan la conducta del individuo.

\section{MÉTODO}

Esta investigación obedece a un enfoque cualitativo y descriptivo que permite reconocer y analizar los fenómenos sociales desde una perspectiva abierta, naturalista y con énfasis en las percepciones y vivencias de los participantes (en este caso de mujeres en situación de violencia de las veredas Boquerón, El Carmen y Frutillo, del municipio de Ventaquemada, en Boyacá).

Las veredas fueron seleccionadas debido a que dentro del contexto se han normalizado los actos de violencia hacia la mujer, lo que ha sido un causante de desconocimiento sobre esta problemática y de que gran parte de los casos no hayan sido denunciados, dadas las dinámicas patriarcales presentes en el contexto ventaquemense. A raíz de esto resulta valioso realizar un análisis de las vivencias de estas mujeres para así fortalecer los procesos de apoyo e intervención psicosocial que diferentes entidades pueden ofrecer en este medio.

En cuanto a la selección de la muestra, está compuesta por quince mujeres en situación de violencia, pertenecientes a las veredas Boquerón, El Carmen y Frutillo, seleccionadas mediante un muestreo por redes gracias al apoyo de informantes claves de la comunidad. A estas mujeres les fueron aplicadas una encuesta social, dirigida por una guía de treinta preguntas abiertas y cerradas, y una entrevista semiestructurada, que permitió dar respuesta a aquellas dudas que iban surgiendo a medida que se desarrollaba el dialogo; estas fueron diseñadas con la tabla de categorías, presentadas y validadas por consenso de expertos y referentes teóricos e investigativos previos, con el fin de identificar los factores de riesgo y tipos de violencia presentes en este contexto.

Las categorías de análisis definidas para esta investigación (basadas en la revisión teórica y el estado del arte estructurado) se presentan en la tabla 1. 
Tabla 1. Tabla de categorías de análisis de la investigación.

\begin{tabular}{|c|c|c|}
\hline OBJETIVOS & CATEGORÍAS & SUBCATEGORÍAS \\
\hline \multirow{8}{*}{$\begin{array}{l}\text { Identificar los factores de riesgos que se } \\
\text { presentan en la violencia contra la mujer en } \\
\text { las veredas Boquerón, El Carmen y Frutillo } \\
\text { del municipio de Ventaquemada (Boyacá) }\end{array}$} & \multirow[t]{2}{*}{$\begin{array}{l}\text { Factores de riesgo individuales } \\
\qquad(\text { La Espada, 2004) }\end{array}$} & $\begin{array}{l}\text { Participación } \\
\text { Autoestima (según percepción) }\end{array}$ \\
\hline & & Dinámica familiar \\
\hline & \multirow[t]{3}{*}{ Factores de riesgo relacionales } & Grupo de pares \\
\hline & & Comunidad de las veredas \\
\hline & & Ubicación geográfica \\
\hline & \multirow{4}{*}{ Factores de riesgo sociales } & Apoyo percibido de las instituciones \\
\hline & & Nivel educativo \\
\hline & & Aspectos culturales \\
\hline \multirow{4}{*}{$\begin{array}{l}\text { Determinar los tipos de violencia ejercidos } \\
\text { hacia la mujer en el municipio de } \\
\text { Ventaquemada, Boyacá }\end{array}$} & & Física \\
\hline & \multirow{3}{*}{ Tipos de violencia contra la mujer } & Verbal o psicológica \\
\hline & & Económica \\
\hline & & Sexual \\
\hline
\end{tabular}

Fuente: elaboración propia

De acuerdo a las categorías planteadas anteriormente se categorizó la información obtenida en Excel y se realizó una triangulación de la información, la cual consolida los resultados que se discuten a continuación y que se presentan por objetivos.

\section{RESULTADOS}

A partir del primer objetivo -identificar los factores de riesgos que se presentan en la violencia contra la mujer en las veredas Boquerón, El Carmen y Frutillo del municipio de Ventaquemada (Boyacá) -, se resalta que esta problemática social es un fenómeno que se encuentra presente a gran escala en el contexto, puesto que las quince mujeres participantes en el estudio han sido violentadas, a partir de factores y dinámicas que las vulneran desde un marco individual, relacional y social.

Frente el factor individual se identificó que la mujer en el contexto tiene baja participación dentro de los grupos de su comunidad debido a que el municipio les presta pocos medios y recursos para que se asocien. Como lo resaltan las mujeres de la vereda Boquerón al momento de preguntarles su rol comunitario "aquí solo tiene cabida el hombre, el esposo... ijummm! ¿Para qué uno se mete donde no debe si uno se gana problemas?, como a sumercé en algún momento le puede pasar" (mujer 1), esto debido al sistema patriarcal que se desarrolla en la zona, que lleva a que las mujeres sean controladas por su pareja sentimental en sus ámbitos productivos o domésticos, reproductivos y comunitarios (no se debe olvidar que en el último su participación es limitada), esto a pesar de que exista normatividad vigente a nivel internacional y nacional como lo es la Declaración sobre la Eliminación de la Violencia contra la Mujer; la Convención Interamericana para Prevenir, Sancionar y Erradicar la Violencia contra la Mujer, también conocida como Convención de Belem do Para; la Constitución Política de Colombia de 
1991; la Ley 1257 de 2008, o Ley de la Mujer, y la Ley 731 de 2002, entre otras que adoptan normas para prevenir, remediar y sancionar la violencia con el fin de favorecer la calidad de vida y bienestar de la mujer.

A pesar de esto, algunas mujeres se animan a participar en grupos sociales que prestan servicios a la comunidad, como la Junta de Acción Comunal y labores dentro y fuera de la iglesia. Debe tenerse en cuenta que sus esposos cumplen un rol comunitario significativo dentro de los mismos. Ante el nivel de autoestima según la percepción de este género, se identifica que gran parte considera que su autoestima es media y baja, por el trato que reciben de su familia, mayormente de su cónyuge, por las diferentes dinámicas a las que se enfrentan en su cotidianidad y a su vez la inseguridad que presentan frente a su realidad, esto como consecuencia del maltrato físico, psicológico, sexual y económico ejercido hacia ellas.

Las dinámicas anteriormente nombradas se consideran como factores de riesgo relacionales, ya que la familia, grupo de pares y la comunidad de las veredas han normalizado la violencia en el contexto, puesto que la comunicación entre estas instituciones sociales como redes de apoyo para la mujer es mínima, debido a la cultura patriarcal presente y por estereotipo de que la mujer es la encargada de las labores domésticas: "es nuestra obligación desde que nos casamos, mi madre lo dijo cuando me vine a vivir aquí con el aquel" (mujer 2). Esto debido a que socialmente se han determinado los roles en una familia y no se tiene en cuenta el importante rol de la mujer en el contexto.

Ante los factores de riesgo sociales se evidenció que el nivel educativo juega un papel importante al momento de que la mujer decida participar y participe en grupos sociales. Este ha sido un alto determinante de violencia contra la mujer, ya que de las quince participantes de la investigación, se identificó que trece de ellas no tienen ningún nivel educativo o adelantaron sus estudios hasta primaria. El factor es alarmante puesto que la escolarización es importante en este contexto para así tener mayor conocimiento de los derechos que se tienen y de los mecanismos o redes de apoyo a los que pueden acudir en el caso de que exista vulneración.

Otra dinámica que determina el bajo nivel educativo en la zona es la de los roles adoptados en la familia. Al preguntar a las mujeres por qué decidieron abandonar la escuela, surgen respuestas como esta: "me salí de estudiar por ayudar en la casa, como soy la hija mayor yo hacía los oficios y ayudaba con las cosas de la casa... eso no tenía tiempo para estudiar y así fue que solo llegue a tercero de primaria, además porque no había plata para los cuadernos... que para los lapice y eso, así que yo me quedaba en la casa o acompañaba a mis sagrados padres" (mujer 3). Esto se evidencia altamente en las zonas rurales o en familias que no cuentan con los recursos suficientes para satisfacer sus necesidades básicas.

Cabe resaltar que el escaso nivel educativo es un factor que contribuye a que los hogares no cuenten con los recursos mínimos para tener una buena calidad de vida y que su estrato socioeconómico sea bajo, debido a que en muchos casos la mujer no cuenta con los requerimientos para acceder a oportunidades laborales. Del mismo modo es relevante señalar que sin importar el estrato o credo cualquier mujer está expuesta a 
ser víctima de violencia, pero a pesar de esto se determinó que las mujeres de estrato socioeconómico 1 son más propensas a ser agredidas debido a su dependencia y su poca participación en el medio, generadas por los factores anteriormente nombrados.

De acuerdo al segundo objetivo -determinar los tipos de violencia ejercidos hacia la mujer en el municipio de Ventaquemada (Boyacá) -, se resalta que todas las mujeres participantes alguna vez han experimentado violencia física, psicológica o verbal, sexual y económica, esto debido a la baja participación, liderazgo, autoestima, nivel educativo, nivel socioeconómico y la normalización de la violencia en el contexto, además de los factores de riesgo individuales, relacionales y sociales.

Al reconocer los tipos de violencia existentes en las veredas Boquerón, El Carmen y Frutillo, se determinan algunas de las consecuencias que han traído cada una de ellas en la calidad de vida de la mujer. En cuanto a la violencia física, psicológica y sexual, algunas mujeres relatan sobre qué consecuencias han dejado las agresiones:

"cuando me dan la juetera me dejan unos moratones que me toca tapármelos con la ropa y unos dolorcitos por ahí, además relatan que me dio depresión, eso cuando uno se siente muy triste y perdí una pierna y un dedo de un golpazo que el indio ese me pego, como me llevaron al doctor pude decir que pasó, pero pues en ese momento no hicieron nada" (mujer 4)

Esta problemática de violencia cada vez aumenta más y no se han tomado las medidas adecuadas para intervenir de una manera eficiente en el caso, por falta de conocimiento hacia las redes de apoyo o acompañamiento psicosocial que se desplace en el municipio y dentro de las organizaciones como lo es la comisaria de familia e incluso estaciones de policía u otros sitios estratégicos.

Gran parte de las mujeres no recurren a nadie en busca de red de apoyo, por el hecho de "evitar el chisme y para que mi familia no resulte involucrada en algo mío" (mujer 5), mientras que algunas de ellas dan a conocer su situación a algún familiar, amigo o vecino. Cuando el nivel de violencia es muy alto han pensado en acudir a la policía pero, como lo narran algunas mujeres "es difícil porque no tengo la plata para ir hasta el pueblo, no tengo ni minutos... ehhh y por aquí es difícil ver la policía" (mujer 6). También por algunos condicionantes y amenazas que hace su cónyuge hacia la mujer.

Las mujeres que dependen económicamente de sus esposos, se ven obligadas a cumplir las condiciones que ellos les imponen a la hora del manejo del dinero, asimismo son víctimas de humillaciones cuando sus victimarios les reprochan sus aportes monetarios a la familia, sin tener en cuenta que la mujer en muchos casos participa en el ámbito productivo. Esta subordinación también está dada en el contexto: "mi esposo no me deja trabajar porque solo sirvo para hacer el aseo y cuidar los chinos" (mujer 7). Esta dinámica se repite en el medio debido a la falta de empoderamiento y seguridad existente. Otro factor en este caso social es la falta de oportunidades laborales ya que la mayoría no cuenta con un alto nivel de estudios o ya son excluidas por las mismas dinámicas contextuales.

De acuerdo a los diferentes tipos de violencia contra la mujer presentes se identifica que estos actos se presentan generalmente cuando el agresor se encuentra bajo los 
efectos del alcohol, ya que a nivel departamental y local se reconoce esta zona por el alcoholismo en un marco de cultura patriarcal.

\section{CONCLUSIÓN}

En conclusión, la normalización de la violencia contra la mujer en las veredas Boquerón, El Carmen y Frutillo del municipio de Ventaquemada se determina como uno de los factores de riesgo más relevantes, ya que esto da paso a que se desconozcan las líneas de atención o redes de apoyo con las que cuentan las mujeres en el caso de que en el medio se desarrolle algún tipo de violencia o vulnerabilidad hacia ellas. Por lo tanto es necesario un compromiso no solo de la Alcaldía del municipio, sino también desde la labor del trabajador social, con el fin de dar a conocer estos factores y generar empoderamiento, a partir de la teoría ecológica de los sistemas.

El nivel socioeconómico de la mujer no es un determinante directo para ser agredida por actores de su medio, aunque cabe resaltar que donde mayormente se presenta violencia es en los estratos socioeconómicos bajos. Además, las mujeres de Ventaquemada presentan un índice de violencia considerable, lo que ha hecho que la violencia física y psicológica prevalezca, afectando así el desarrollo integral, bienestar y calidad de vida de este género en el medio.

La violencia contra la mujer puede estar categorizada en violencia física, psicológica, sexual y económica dándose en los contextos de pareja, familia o sujetos pertenecientes a las redes de apoyo cercanas, es decir que pertenezcan al microsistema de la mujer, que como consecuencias ha generado que el género femenino esté inmerso en dinámicas de discriminación, falta de oportunidades, desigualdad, inequidades y falta de espacios para que la posibilidad de ejercer otras habilidades o capacidades en pro y beneficio de la sociedad, tal como lo han venido haciendo en los ámbitos reproductivos o domésticos, productivos y comunitarios, los cuales se han invisibilizado por la cultura que predomina en el medio.

Es importante señalar que la violencia contra la mujer es una problemática influenciada por el medio, tal como se evidenció en las veredas abordadas del municipio de Ventaquemada, donde se identificó la cultura machista y patriarcal que ha determinado estereotipos e imaginarios de la mujer sobre su importante rol en el medio. Se suma a esto que en dicho contexto no existía ninguna política pública sobre este fenómeno social, a pesar de que se ejecute normatividad a nivel internacional, nacional y departamental.

La violencia contra la mujer es una problemática compleja de erradicar, pero se podría intervenir desde propuestas e iniciativas desde trabajo social, con el fin de que los índices disminuyan, ya que es alarmante que en estas veredas (veredas Boquerón, El Carmen y Frutillo) se normalice el fenómeno, a pesar de las consecuencias no solo a nivel individual, sino también social que se han visibilizado en la mayoría de los casos de violencia. Es por esto que desde esta profesión y disciplina fue importante, a partir de las iniciativas, desarrollar campañas de información con el fin de que la sociedad ventaquemense adopte en conjunto una actitud activa en la solución de este problema y denuncie todas las acciones de violencia para que la erradicación de esta sea progresiva. Además, se dieron bases y hubo participación en la creación de la política pública de equidad de género, enfocada en la violencia contra la mujer y, por consiguiente, programas de empoderamiento e incentivación del rol de la mujer en los diferentes ámbitos. 


\section{REFERENCIAS}

Acosta, M. (2015). Comportamiento de la violencia de pareja. Colombia. Fundación Ideas para la Paz. Recuperado de: http://www.medicinalegal.gov.co/documents/88730/3418 907/5.+VIOLENCIA+DE+PAREJA.pdf/03de1fd1-092d-495f-99d3-1aacee2991c6

Alvarado, W. (2016). Informe del evento de violencia de género en Boyacá, hasta el periodo epidemiológico VI, Boyacá, 2016. Tunja: Grupo de Vigilancia en Salud Pública.

Bronfenbrenner, U. (1987). La ecología del desarrollo humano. Barcelona: Paidós.

Cardona, J. (2012). La violencia contra la mujer: estado del arte en materia de prevención, atención y sanción. Bogotá: Universidad piloto de Colombia. Recuperado de: http:// www.contextos-revista.com.co/Revista\%209/A2 Mujer\%20derechos.pdf

Comisión Nacional para Prevenir y Erradicar la Violencia contra las Mujeres. (2010). Glosario de términos sobre violencia contra la mujer. México D.F.: Editorial Pax. Recuperado de: http://www.gob.mx/cms/uploads/attachment/file/79365/Glosario de Terminos completo 1 .pdf

Corporación Humanas (2008, junio). La situación de las mujeres víctimas de violencias de género en el sistema penal acusatorio. Recuperado de: http://perso.unifr.ch/ derechopenal/assets/files/obrasportales/op 20110607 01.pdf

DANE y Gobernación de Boyacá (2016). Plan Departamental de Desarrollo de Boyacá, Creemos en Boyacá, tierra de paz y libertad - 2016-2019.

Fuente, M. y Morán, R. (2011). Raíces profundas. La violencia contra las mujeres. Madrid: Ediciones Polifemo.

Gallardo, Y.; Gallardo, R.L.; Núñez, M.A. y Varela, M.E. (2009). Caracterización de la violencia intrafamiliar en la mujer. Media Luna. Revista Habanera de Ciencias Médicas, 8(5), 131141. Recuperado de: http://scielo.sld.cu/pdf/rhcm/v8s5/rhcm18509.pdf

Gómez, C.; Murad, R. y Calderón, M.C. (2013). Historias de violencia, roles, prácticas y discursos legitimadores. Violencia contra las mujeres en Colombia 2000-2010. Recuperado de: https://www.minsalud.gov.co/sites/rid/Lists/BibliotecaDigital/RIDE/ INEC/INV/7\%20\%20VIOLENCIA $\% 20$ CONTRA $\% 20$ LAS $\% 20 M U J E R E S \% 20 E N \% 20$ COLOMBIA.pdf

Guedes, A.; García, C. y Bott, S. (2014). Violencia contra las mujeres en Latinoamérica y el Caribe. Foreign Affairs Latinoamérica, 14(1), 41-48. Recuperado de: http://www2. paho.org/hq/index.php?option $=$ com docmanytask $=$ doc viewyItemid $=270 y g i d=2415$ 7ylang=es

Jiménez, F. (2012). Conocer para comprender la violencia: origen, causas y realidad. Convergencia, Revista de Ciencias Sociales, 58, enero-abril, 13-22. Recuperado de: http://www.scielo.org.mx/pdf/conver/v19n58/v19n58a1.pdf 
Kail, R. y Cavanaugh, J. (2011). Desarrollo humano. Una perspectiva del ciclo vital. México: Cengage Learning Editores, S.A.

La Espada, M.; Iraurgí, L y Arostegi, E. (2004). Factores de riesgo y de protección frente al consumo de drogas: hacia un modelo explicativo del consumo de drogas en jóvenes de la CAPV. Deusto, España: Instituto Deusto de Drogodependencias, Universidad de Deusto. Recuperado de: http://www.izenpe.eus/s154812/es/contenidos/informacion/ publicaciones ovd inf txostena/es 9033/adjuntos/informe txostena14.pdf

Miranda, C.F.; Santos, D.J. y Pattaro, F. (2015). Violencias en la posmodernidad: resistencias, paradigmas y conflictos en Latinoamérica. Barranquilla: Editorial Coruniamericana.

Naciones Unidas. (1993). Declaración sobre la Eliminación de la Violencia contra la Mujer. Nueva York. Recuperado de: http://www.who.int/es/news-room/fact-sheets/detail/ violence-against-women

Organización Mundial de la Salud. (2013). Estimaciones mundiales y regionales de la violencia contra la mujer: prevalencia y efectos de la violencia conyugal y de la violencia sexual no conyugal en la salud. Departamento de Salud Reproductiva e Investigación. Recuperado de: http://apps.who.int/iris/bitstream/handle/10665/85243/WHO RHR HRP 13.06 spa.pdf;jsessionid=72AA71A6AC13271A4BAAA3047684CF52? sequence $=1$

Pérez, M.; Barreto, P.; Roa, M. y Rubiano, G. (2015). Percepción de riesgo y factores asociados al consumo de drogas legales e ilegales en estudiantes de la Universidad de Boyacá. Revistas digitales Universidad de Boyacá, 1(2), 83-102. Recuperado de: http://revistasdigitales.uniboyaca.edu.co/index.php/EFQ/article/view/167/161

Riveros, S. (2014). Violencia en Boyacá: fenómeno que siempre ha existido y se ha mantenido oculto. Troncando Sin Fronteras Boyacá. Recuperado de: https://trochandosinfronteras. info/feminicidios-boyaca-fenomeno-siempre-ha-existido-se-ha-mantenido-oculto/

Rodríguez, D. (2013). Caracterización psicosocial de mujeres víctimas de violencia conyugal de la comisaría de familia del municipio de Ramiriquí, Boyacá, año 2011-2012. [Trabajo de grado para optar al título de psicóloga]. Tunja: Universidad Nacional Abierta y a Distancia. Recuperado de: http://stadium.unad.edu.co/preview/UNAD.php?url=/ bitstream/10596/2267/1/23965790.pdf

United Nations. Secretary-General's Campaign. Unite to end violence against women. (2009, Noviembre). Violencia contra las mujeres. La situación. Recuperado de: http://www. un.org/es/events/endviolenceday/pdfs/unite the situation sp.pdf

Yugueros, A.J. (2014). La violencia contra las mujeres: conceptos y causas. Barataria, Revista Castellano-Manchega de Ciencias Sociales, 18, enero-diciembre, 147-159. Recuperado de: http://www.redalyc.org/pdf/3221/322132553010.pdf 